\title{
The Activation Strain Tensor: Nonhydrostatic Stress Effects on Crystal-Growth Kinetics
}

\section{Citation}

Aziz, Michael J., Paul C. Sabin, and Guo-Quan Lu. 1991. The Activation Strain tensor:

Nonhydrostatic stress effects on crystal growth kinetics. Physical Review B 44(18): 9812-9816.

\section{Published Version}

http://dx.doi.org/doi:10.1103/PhysRevB.44.9812

\section{Permanent link}

http://nrs.harvard.edu/urn-3:HUL.InstRepos:2860451

\section{Terms of Use}

This article was downloaded from Harvard University's DASH repository, and is made available under the terms and conditions applicable to Other Posted Material, as set forth at http:// nrs.harvard.edu/urn-3:HUL.InstRepos:dash.current.terms-of-use\#LAA

\section{Share Your Story}

The Harvard community has made this article openly available.

Please share how this access benefits you. Submit a story.

Accessibility 


\title{
The activation strain tensor: Nonhydrostatic stress effects on crystal-growth kinetics
}

\author{
Michael J. Aziz, Paul C. Sabin, and Guo-Quan Lu* \\ Division of Applied Sciences, Harvard University, Cambridge, Massachusetts 02138
}

(Received 1 July 1991)

\begin{abstract}
The solid-phase epitaxial-growth rate of crystalline $\mathrm{Si}$ from the amorphous $\mathrm{Si}$ on the tensile side is greater than on the compressive side of elastically bent wafers, in marked contrast to the behavior observed under hydrostatic pressure. The phenomenology of an activation strain tensor, the nonhydrostatic analogue of the activation volume, is developed to characterize such measurements. The measurement permits us to characterize to first order the entire activation strain tensor for solid-phase epitaxy of $\mathrm{Si}(001)$ : The transition state for this process involves an in-plane expansion and a contraction in the direction normal to the interface. Its symmetry is inconsistent with all proposed bulk point-defect mechanisms.
\end{abstract}

Nonhydrostatic stresses are common during crystal growth. They can arise in polycrystalline materials from anisotropic elasticity or thermal expansion, or during recrystallization, ${ }^{1}$ precipitation, or other solid-state transformations. Even in single crystals, they generally occur during strained-layer heteroepitaxy or any other epitaxial growth process involving composition discontinuities or gradients. The effect of nonhydrostatic stress on the energetics of phase transformations is currently an area of active research, ${ }^{2,3}$ but its effect on the interfacial or atomic mobilities themselves has not been addressed. Although the effect of pressure on the kinetic rate constants for crystal growth have been studied, ${ }^{4-6}$ a measurement of the effect of nonhydrostatic stresses has proved elusive. This is due in part to the difficulty of imposing a controlled amount of nonhydrostatic stress without having it partially relieved and rendered, on a fine scale, nonuniform by dislocation injection.

Fratello, Hays, and Turnbull found evidence suggesting that nonhydrostatic stresses were even more effective in enhancing quartz growth than was pressure, ${ }^{4}$ but they were unable to control or quantify the former effect. Similarly, the solid-phase epitaxial-growth (SPEG) rate of crystalline $\mathrm{Si}(c-\mathrm{Si})$ into self-implanation-amorphized $\mathrm{Si}$ ( $a$-Si) overlays is also enhanced by pressure. ${ }^{6}$ Here we report measurements for the effect of nonhydrostatic stress on this rate, a theory for its consequences on an atomistic scale, and a comparison to a class of atomistic models for the SPEG process. The measurement was accomplished by elastically bending Si wafers over fused quartz rods at a temperature too low for plastic deformation to occur but high enough for SPEG to proceed at measurable rates. The difference between the growth rates on the compressive and tensile sides of the wafer was measured, as was the behavior of the growth rate as the stress varied along the length of the wafer.

Si(001) wafers ( $p$ type, $1 \Omega \mathrm{cm}, 0.84 \mathrm{~mm}$ thick, polished on both sides) were implanted on both sides at $77 \mathrm{~K}$ with ${ }^{30} \mathrm{Si}^{+}\left(60 \mathrm{keV}, 1 \times 10^{15} / \mathrm{cm}^{2}\right.$, and $\left.180 \mathrm{keV}, 2 \times 10^{15} / \mathrm{cm}^{2}\right)$ to create amorphous surface layers $2800 \AA$ thick. Wafers were diced into bars $>20 \mathrm{~mm}$ long in the [110] direction, by $5 \mathrm{~mm}$ wide.

Stress was imposed in air with a three-point bending system, depicted in Fig. 1. The sample rested on a pair of parallel fused quartz rods spaced $20 \mathrm{~mm}$ apart and set into grooves in a circular brass base plate. A third fused quartz rod made contact with the sample from above. Weights were added to the top of the upper plate to control the stress applied to the sample. The entire apparatus fit snugly into a vertical cylindrical furnace. A Chromel-Alumel thermocouple was in contact with the sample. An unstressed "calibration sample," which had only the top surface implanted, lay below the stressed sample; it served to calibrate temperatures and/or gradients.

The apparatus was pre-heated in the furnace to $\sim 560^{\circ} \mathrm{C}$. The top piece of the apparatus was removed and the sample and "calibration sample" were inserted; this resulted in a temperature drop of slightly more than

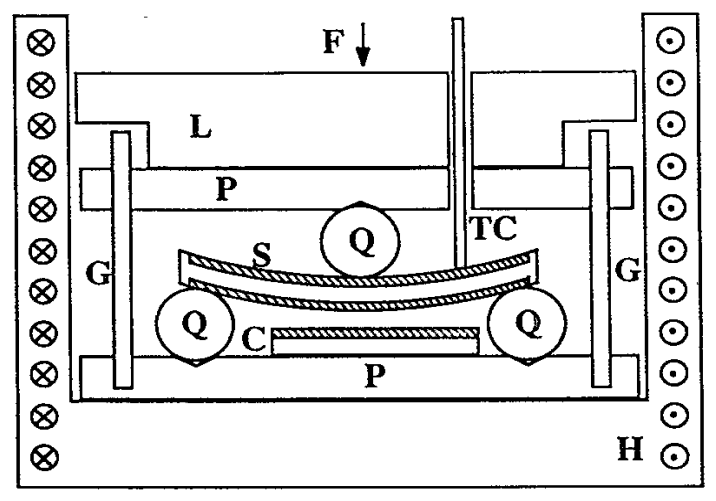

FIG. 1. Schematic cross section of three-point bending apparatus for annealing wafers under nonhydrostatic stress. S: sample, cross-hatched areas correspond to amorphous $\mathrm{Si}$; $\mathrm{C}$ : calibration sample; $Q$ : fused quartz support rods; P: brass plate; G: guide posts; TC: thermocouple; $\mathrm{H}$ : heating element; $\mathrm{F}$ : weights; L: lid. 
$20^{\circ} \mathrm{C}$. Subsequently the temperature was stabilized at $540 \pm 2^{\circ} \mathrm{C}$ within $8 \mathrm{~min}$. Concurrently, the appropriate weight was completely added within $4 \mathrm{~min}$. Typical anneal durations were 60-90 min. The sample was removed from the furnace immediately after the load was lifted. After annealing, the thickness of the remaining $a-$ $\mathrm{Si}$ layer was determined by Rutherford backscattering spectrometry and ion channeling. ${ }^{7}$ The beam spot diameter was $\sim 1.5 \mathrm{~mm}$.

The stress state of the bar-shaped wafer in bending can be approximated as a uniaxial stress that varies linearly through the wafer thickness, with one side in compression and the other side in an equal amount of tension. Under the three-point bending load, the stress in the wafer also varies linearly from the single loading point at the center silica rod to the points of contact of the two supporting end rods. The magnitude of the stress is maximum on the wafer surface at the central loading point, and is zero at the point of contact of the two supporting end rods. At the wafer surface,

$$
\sigma_{11}(x)= \pm \frac{|x|-L / 2}{b h^{2} / 3} F,
$$

where $\sigma_{i j}$ is the stress tensor, $x$ is the distance from the point of contact of the center rod, $L$ is the length of the bar between the supporting end rods, $F$ is the loading force on the bar, $b$ is the width, and $h$ is the thickness of the bar. All other $\sigma_{i j}$ are zero in this approximation. The stress calculated from Eq. (1) exists in the crystal at the crystalline-amorphous interface. There is no stress in the bulk of the $a$-Si due to stress relief by viscous flow. ${ }^{8}$

The growth rate for a sample whose maximum stress was $\sigma_{11}=6 \mathrm{kbar}$ during an anneal at $540^{\circ} \mathrm{C}$ for $76 \mathrm{~min}$ is shown in Fig. 2(a). The tensile side (dashed curve) grew faster and the compressive side (solid curve) of this wafer grew slower than did either side of two "control samples" (circles and triangles), which were annealed at low stress on separate runs in the same loading configuration as the high stress samples (note: these are different from the "calibration samples"). The load on the control samples was not reduced all the way to zero, in order to maintain the same thermal contact between wafer and silica rods as in the high-stress sample. The growth rates on the tensile and compressive sides approach each other as $x= \pm 10 \mathrm{~mm}$, where the stress vanishes, is approached. In Figs. 2(b) and 2(c) we show similar results for different wafers stressed to $6 \mathrm{kbar}$ for $70 \mathrm{~min}$ and $5 \mathrm{kbar}$ for 60 min, respectively. In all cases, the difference between the growth rates on the tensile and compressive sides is maximum in the center of the wafer, where the stress difference is maximum; and no such effect is observed for the control samples, which were exposed to an identical thermal environment.

The failure of the growth rates on the tensile and compressive sides to surround symmetrically the growth rates in the control samples is probably due to a small sample-to-sample variation in anneal temperature. However, even if there had been no control samples, the temperature difference between two sides of any particular wafer is far too small to be invoked to account for the difference in growth rates. ${ }^{9}$ It is also possible that the $a$ -
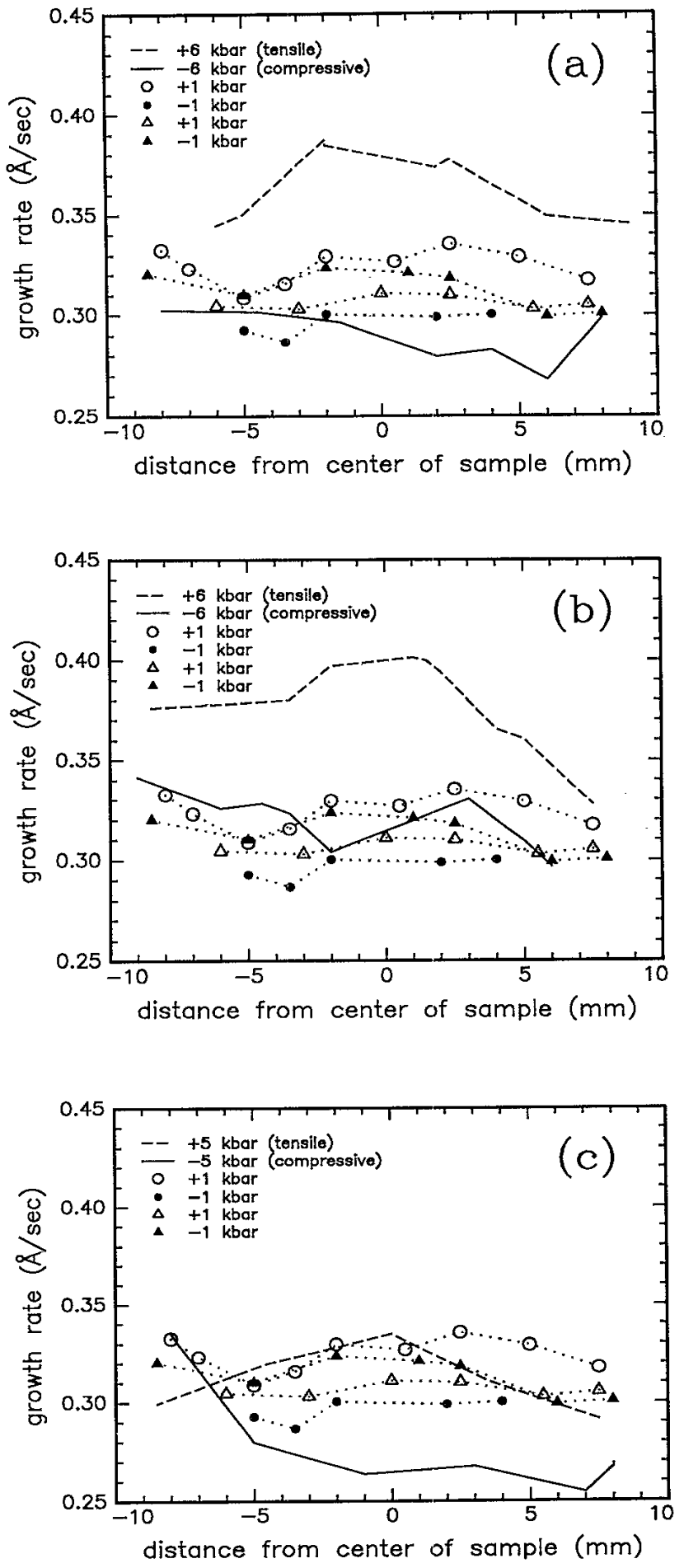

FIG. 2. Variation of average solid phase epitaxial growth rate at $540^{\circ} \mathrm{C}$ with stress along length of bent wafer. Dashed line: tensile side of specimen; solid line: compressive side. In each case the magnitude of $\sigma_{11}$, indicated in the figure, is maximum in the center $(x=0)$, and varies linearly to zero at the ends $(x= \pm 10 \mathrm{~mm})$. Dotted lines connect points taken from "control" samples under minimal load. Circles correspond to growth on opposite sides on of one control sample; triangles to opposite sides of another. Differences between tensile and compressive sides of high-stress samples are greatest in the center, where the stress difference is maximum, and lowest at the ends, where stresses vanish. 
Si layer becomes thinner on the tensile side, and thicker on the compressive side, by flow. The observed effect is over three times too large to be due to flow alone, even if there is sufficient flow to completely relax all stresses in the $a-\mathrm{Si}$.

All data from Fig. 2 are divided by the best-fit zerostress velocity for each sample, and plotted in Fig. 3 to display the effect of applied stress on the velocity. The curve fitted to the data is derived from an extension of transition-state theory (TST) to nonhydrostatic stress states, as described below.

The constant activation energy ${ }^{10}$ and activation volume $^{6}$ of the SPEG rate of Si indicate that TST is an appropriate basis for the description of this process. According to $\mathrm{TST}^{11}$ the transition rate is proportional to the probability of a fluctuation from the starting state $a$ to the transition state $a^{*}$. The atomistic nature of the transition state for the rate-limiting step of the SPEG process is still a matter of debate. ${ }^{12,13}$ For TST, it need only be identified as a thermodynamic subsystem in thermal and mechanical contact with a solid reservoir. From a battery of measurements the energy, volume, etc., of the transition state, relative to the starting state, have been deduced through TST.

Nonhydrostatic stress states affect the thermodynamics of the system, altering the probability of a fluctuation to the transition state. The generalization of TST to nonhydrostatic stresses is not straightforward because the chemical potential is not defined within a nonhydrostatically stressed solid. ${ }^{1,2}$ However, Landau and Lifshitz treat the appropriate fluctuations for fluids under constant hydrostatic pressure ${ }^{14}$ without using the chemical potential, by considering the equilibrium fluctuations of

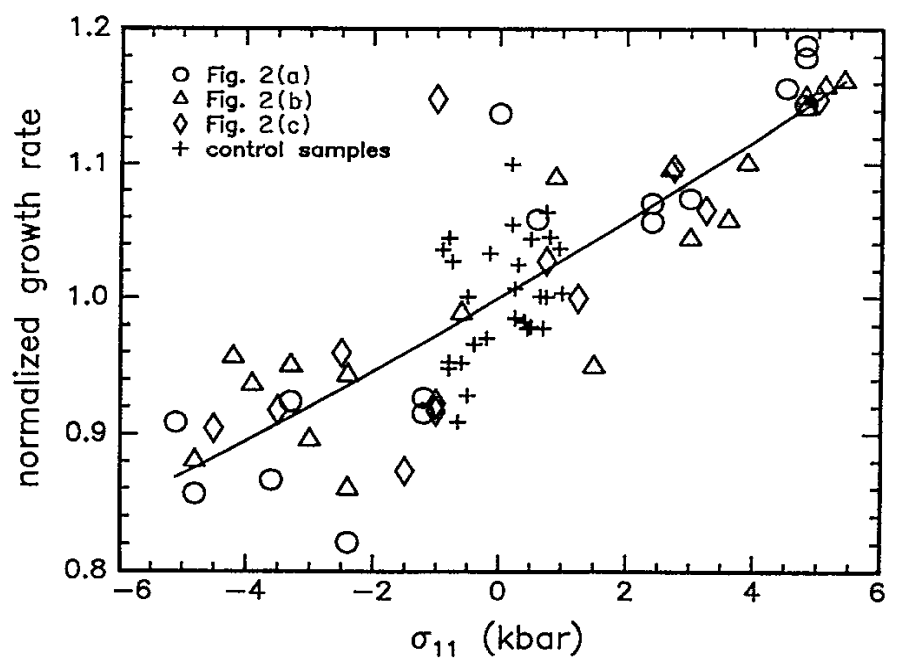

FIG. 3. Variation of growth rate with applied stress for all samples. Open symbols correspond to high-stress samples in Fig. 2. Scatter in data can be reduced by taking into consideration controls and the trends evident from Fig. 2, but such manipulations are not necessary to obtain an approximate magnitude for the stress effect. Curve is fit to $\mathrm{Eq}$. (7) with $\Delta V_{11}^{*}=0.15 \Omega$. the thermodynamic variables in a closed system. Here we generalize to nonhydrostatic conditions.

The development of TST under nonhydrostatic conditions must be performed with some care because, (i) strain, per se, is not conserved in a closed system in the same way that volume is conserved, (ii) the small strain approximation may not be valid, (iii) stresses in a solid reservoir, unlike the pressure in a large fluid reservoir, are neither constant nor spatially uniform during the transition $a \rightarrow a^{*}$, and (iv) stresses are not necessarily continuous across the subsystem-reservoir boundary.

In the $a \rightarrow a^{*}$ fluctuation, the subsystem undergoes a chemical change to a configuration with a different size and shape. This same change may be brought about by an external agent performing mechanical work $R$ on the subsystem. The growth velocity $v$ is proportional to the fluctuation "rate, which is proportional to $\exp \left(-R_{\min } / k T\right)$. Here $R_{\min }$ is the minimum value of $R$, done on a reversible path, and $k T$ has the usual meaning.

We wish to determine $\partial R_{\min } / \partial \sigma_{i j}$. This can be done using Rice's thermodynamic formalism ${ }^{15}$ for solids with internal variables such as the position of a dislocation or, in our case, the shape change of a small internal volume element (the "subsystem") surrounding the atoms involved in the fluctuation from $a$ to $a^{*}$. We label the macroscopic, externally applied stress field $\sigma_{i j}^{\mathrm{mac}}$ and the macroscopic strain of the sample $\varepsilon_{i j}^{\text {mac }}$. The strain in the subsystem is not necessarily small, necessitating the use of the deformation gradient $e_{i j}^{\mathrm{sub}} \equiv \partial u_{j} / \partial x_{i}$, where $\mathbf{u}$ is the displacement field. The stresses on the boundaries of the subsystem are $T_{i j}^{\mathrm{sub}}\left(\sigma_{i j}^{\mathrm{mac}}, e_{i j}^{\mathrm{sub}}\right)$, where $T_{i j}$ (the first PiolaKirchhoff stress) is the ratio of actual force to original, undeformed area. $R_{\min }$ is obtained from the product of the forces and the displacements on the boundaries of the subsystem during the transition $a \rightarrow a^{*}$ :

$$
R_{\min }=V_{0}^{\mathrm{sub}} \int_{a}^{a^{*}} T_{i j}^{\mathrm{sub}}\left(\sigma_{i j}^{\mathrm{mac}}, e_{i j}^{\mathrm{sub}}\right) d e_{i j}^{\mathrm{sub}},
$$

where $V_{0}^{\text {sub }}$ is the volume of the subsystem in state $a$. The stress derivatives of $R_{\min }$ are then given by

$$
\frac{\partial R_{\min }}{\partial \sigma_{i j}^{\operatorname{mac}}}=V_{0}^{\mathrm{sub}} \int_{a}^{a^{*}} \frac{\partial}{\partial \sigma_{i j}^{\mathrm{mac}}} T_{i j}^{\mathrm{sub}} d e_{i j}^{\mathrm{sub}} .
$$

We introduce $\Phi$, Rice's generalized Helmholz free energy that includes the work done by internal variables. At constant temperature it is defined by

$$
d \Phi=V_{0}^{\mathrm{mac}} \sigma_{i j}^{\mathrm{mac}} d \varepsilon_{i j}^{\mathrm{mac}}+V_{0}^{\mathrm{sub}} T_{i j}^{\mathrm{sub}} d e_{i j}^{\mathrm{sub}},
$$

where $V_{0}^{\text {mac }}$ is the sample volume. From its dual potential on strain, $\Psi \equiv \Phi-V_{0}^{\mathrm{mac}} \sigma_{i j}^{\mathrm{mac}} \varepsilon_{i j}^{\mathrm{mac}}$ follows the Maxwell relation

$$
V_{0}^{\mathrm{sub}} \frac{\partial T_{i j}^{\mathrm{sub}}}{\partial \sigma_{i j}^{\mathrm{mac}}}=-V_{0}^{\mathrm{mac}} \frac{\partial \varepsilon_{i j}^{\mathrm{mac}}}{\partial e_{i j}^{\mathrm{sub}}} .
$$

Substitution into (3) yields

$$
\frac{\partial R_{\min }}{\partial \sigma_{i j}^{\text {appl }}}=-\Delta V_{i j}^{*},
$$

where $\Delta V_{i j}^{*} \equiv V_{0}^{\mathrm{mac}} \varepsilon_{i j}^{*, \mathrm{mac}}$, and $\varepsilon_{i j}^{*, \mathrm{mac}} \equiv \int_{a}^{a *} d \varepsilon_{i j}^{\mathrm{mac}}$ is the ac- 
tivation strain tensor, which takes the sample from the configuration $a$ to $a^{*}$. Hence,

$$
v\left(\sigma_{i j}^{\mathrm{mac}}\right)=v(0) \exp \left[\frac{\sigma_{i j}^{\mathrm{mac}} \Delta V_{i j}^{*}}{k T}\right] .
$$

The above derivation assumed the strain to be uniform over the subsystem. We do not prove it here, but Eq. (7) still holds without this assumption.

For the purposes of model building, it would also be instructive to know how the microscopic shape change of the subsystem determines the stress dependence of $v$. From classical continuum elasticity theory, under certain conditions we will have

$$
V_{0}^{\mathrm{mac}} \varepsilon_{i j}^{*, \mathrm{mac}}=V_{0}^{\text {sub }} t_{i j}^{* \text {,sub }} \text {, }
$$

where $t_{i j}^{*}$ is the "transformation strain" of the subsystem, ${ }^{16}$ i.e., the shape change of the subsystem during the transition $a \rightarrow a^{*}$ in the absence of the constraining effects of the surrounding solid material. Equation (8) holds if strains are small, the elastic constants are the same in the subsystem and in the surroundings in both states $a$ and $a^{*}$, and no sliding and no gaps occur at the boundary of the subsystem.

The curve in Fig. 3 is a fit to Eq. (7). We find $\Delta V_{11}^{*}=(0.15 \pm 0.01) \Omega$, where $\Omega$ is the atomic volume of $c$-Si. Since symmetry requires that $\varepsilon_{22}^{*}=\varepsilon_{11}^{*}$, and since we have previously measured ${ }^{6}$ the activation volume (the trace of $\Delta V_{i j}^{*}$ ) to be $\Delta V^{*}=-0.28 \Omega$, we have $\Delta V_{33}^{*} / \Omega=-0.58$. Hence the transition state involves an in-plane expansion and a contraction in the direction normal to the interface. ${ }^{17}$ From this we predict that (i) biaxial stress of the type normally encountered in heteroepitaxy will have twice the effect of uniaxial stress; (ii) uniaxial compression in the direction normal to the interface will enhance the growth rate even more than does hydrostatic pressure. This explains the excessive magnitude of the apparent $\Delta V^{*}$ measured in a piston-cylinder apparatus, in which a nonhydrostatic component of just this type might be expected, in an early experiment. ${ }^{7}$

By comparing SPEG and crystal self-diffusion rates we ruled out models in which thermal generation and motion to the interface of point defects from the bulk of the crystal were the rate-limiting steps for crystal growth. ${ }^{6}$ The shape of the measured activation strain tensor is inconsistent with the formation or motion of any point defect in the bulk of either phase. With these mechanisms, the measured activation strain tensor is the sum of a strain of defect formation and a strain of defect migration, and the measurement yields an average over many defects during the growth of many monolayers of material. The average strain of point-defect formation must be isotropic in amorphous phases and in cubic crystals. The strain of migration, however, need not be isotropic. Consider, for example, tension in the plane of the interface opening up more space for atoms to migraie toward the interface, enhancing their mobility in the [001] direction only. This would be consistent with our observation of in-plane tension-enhanced growth rates. However, in this case hydrostatic pressure will correspondingly reduce that space and consequently reduce the mobility, which is contrary to our observation of pressure-enhanced growth rates. Furthermore, creep measurements ${ }^{8,18}$ indicate that the stresses should be fully relaxed in the amorphous phase in our experiments. For bulk amorphous point-defect mechanisms under these circumstances, wafer-bending cannot affect the growth rate at all.

These results imply that a substantial contribution to the rate-limiting step for the growth process must come from defect formation or motion at the $c$-a interface. They are inconsistent with all bulk point-defect mechanisms proposed to date, ${ }^{13}$ but consistent with proposed interface point-defect mechanisms. ${ }^{12}$ As we have shown ${ }^{19}$ that the Williams-Elliman interfacial kink site mechanism is a special case of the Spaepen-Turnbull interfacial dangling bond mechanism, all proposed mechanisms except one have been eliminated. Point-defect generation in the bulk is not entirely impossible, but it must be accompanied by a significant barrier to motion in or incorporation into the interface. Because the two processes combined in series would have to display a constant activation energy over ten decades in rate, ${ }^{10}$ it seems highly implausible that transport of bulk point defects to the interface plays any role in determining the rate of thermal SPEG.

Crystal symmetry also requires that if there is a single transition state then all nondiagonal elements to the activation strain tensor must be zero for the Si(001) interface. Symmetry-equivalent transition states are possible with, e.g., opposite values of the shear $\varepsilon_{31}^{*}$, in which case $\ln v$ would be parabolic, to lowest order, in $\sigma_{31}$. A measurement of the $\sigma_{31}$ dependence for differently oriented wafers, however, might yield off-diagonal elements.

Note added in proof. The prediction that biaxial stress as normally encountered in heteroepitaxy will have twice the effect of uniaxial stress has been confirmed. By comparing SPEG rates on stressed and stress-related substrates during heteroepitaxy of $\mathrm{Si}_{1-x} \mathrm{Ge}_{x}$ on $\mathrm{Si}$, Hong et al. [Q.-Z. Hong, J. G. Zhu, J. W. Mayer, W. Xia, and S. S. Lau, J. Appl. Phys. (to be published)] find $\Delta V_{11}^{*}=+0.17 \Omega$ for samples under biaxial compression.

We are grateful to K.-R. Lee and J. F. Chervinsky for technical assistance and to J. R. Rice, F. Spaepen, and H. A. Stone for helpful discussions. We thank J. W. Mayer for permission to quote results prior to publication. Samples were implanted at the Surface Modification and Characterization Facility at Oak Ridge National Laboratory. This research was supported by NSF-DMR-8913268. One of us (P.C.S.) was supported jointly by the Undergraduate Faculty Aide Program, the Materials Research Laboratory and the Division of Applied Sciences at Harvard University. 
*Present address: Alcoa Technical Center, Alcoa, PA 15069. ${ }^{1}$ W. B. Kamb, J. Geol. 67, 153 (1959).

${ }^{2}$ F. C. Larché and J. W. Cahn, Acta Metall. 33, 331 (1985).

${ }^{3}$ P. W. Voorhees and W. C. Johnson, J. Chem. Phys. 84, 5108 (1986); Phys. Rev. Lett. 61, 225 (1988); P. H. Leo, W. W. Mullins, R. F. Sekerka, and J. Vinals, Acta Metall. Mater. 38, 1573 (1990).

${ }^{4}$ V. J. Fratello, J. F. Hays, and D. Turnbull, J. Appl. Phys. 51, 4718 (1980).

${ }^{5}$ M. J. Aziz, E. Nygren, J. F. Hays, and D. Turnbull, J. Appl. Phys. 57, 2233 (1985); G. Devaud, M. J. Aziz, and D. Turnbull, J. Non-Cryst. Solids 109, 121 (1989); E. Chason and M. J. Aziz, J. Non-Cryst. Solids 130, 204 (1991).

${ }^{6}$ G. Q. Lu, E. Nygren, M. J. Aziz, D. Turnbull, and C. W. White, Appl. Phys. Lett. 54, 2583 (1989); 56, 137 (1990).

${ }^{7}$ E. Nygren, M. J. Aziz, D. Turnbull, J. M. Poate, D. C. Jacobson, and R. Hull, Appl. Phys. Lett. 47, 232 (1985).

${ }^{8} \mathrm{~A}$. Witvrouw and F. Spaepen, in Kinetics of Phase Transformations, edited by M. J. Aziz, G. B. Stephenson III, and M. O. Thompson, MRS Symposia Proceedings No. 205 (Materials Research Society, Pittsburgh, in press).

${ }^{9}$ The vertical temperature gradient in the 9-mm-high chamber was estimated to be no more than $0.7^{\circ} \mathrm{C} / \mathrm{mm}$ by two independent methods. A calculation of the temperature gradient in the Si wafer, taking into account heat transfer by conduction and radiation and assuming the vertical heat flux to be constant through both the silicon wafer and the surrounding air, indicates that the temperature difference between the tensile and compressive surfaces of the sample is less than $0.2^{\circ} \mathrm{C}$. (The gradient in the air is not large enough for convection to occur.)

${ }^{10}$ G. L. Olson and J. A. Roth, Mater. Sci. Rep. 3, 1 (1988).

${ }^{11}$ S. Glasstone, K. J. Laidler, and H. Eyring, The Theory of Rate Processes (McGraw-Hill, New York, 1948).

${ }^{12}$ F. Spaepen and D. Turnbull, in Laser-Solid Interactions and Laser Processing-1978, Materials Research Society, Boston, Proceedings of the Symposium of Laser-Solid Interactions and Laser Processing, edited by S. D. Ferris, H. J. Leamy, and J. M. Poate, AIP Conf. Proc. No. 50 (AIP, New York, 1979), p. 73; J. S. Williams and R. G. Elliman, Phys. Rev.
Lett. 51, 1069 (1983)

${ }^{13}$ L. Csepregi, R. P. Kullen, J. W. Mayer, and T. W. Sigmon, Solid State Commun. 21, 1019 (1977); I. Suni, G. Goltz, M.-A. Nicolet, and S. S. Lau, Thin Solid Films 93, 171 (1982); J. Narayan, J. Appl. Phys. 53, 8607 (1982); L. E. Mosley and M. A. Paesler, Appl. Phys. Lett. 45, 86 (1984); S. T. Pantelides, in Fundamentals of Beam-Solid Interactions and Transient Thermal Processing, edited by M. J. Aziz, L. E. Rehn, and B. Stritzker, MRS Symposia Proceedings No. 100 (Materials Research Society, Pittsburgh, 1988), p. 387.

${ }^{14}$ L. D. Landau and E. M. Lifshitz, Statistical Physics, 2nd revised English ed. (Pergamon, New York, 1969), pp. 57-60, 348 and 349.

15J. R. Rice, J. Mech. Phys. Solids 19, 433 (1971); J. R. Rice, in Constitutive Equations in Plasticity, edited by A. S. Argon (MIT Press, Cambridge, MA, 1975), Chap. 2, pp. 23-79.

${ }^{16}$ J. D. Eshelby, Proc. R. Soc. London, Ser. A 241, 376 (1957).

${ }^{17}$ The measured $\Delta V_{i j}^{*}$ might be the sum of a formation tensor and a migration tensor, depending on the mechanism.

${ }^{18} \mathrm{P}$. C. Sabin and A. Witvrouw (unpublished) demonstrated that the amorphous phase flows during the course of our experiments at $540^{\circ} \mathrm{C}$ by cooling a sample to room temperature while still under load early in a crystallization run. The wafer remained bent, but straightened out after further annealing with the load removed. Witvrouw and Spaepen (Ref. 8) found that $a$-Si flows at temperatures as low as $150^{\circ} \mathrm{C}$ and studied the rate of change of the viscosity over the temperature range $150-400^{\circ} \mathrm{C}$. They found that the viscosity is a function of thermal history, but its time derviative is not-a sign of bimolecular annihilation kinetics of the defects responsible for viscous flow. Extrapolation of Witvrouw and Spaepen's results to our conditions indicates that after a rapid (1-min) heat-up to $540^{\circ} \mathrm{C}$ the stress in the amorphous phase relaxes to $1 \%$ of its initial value in less than $3 \mathrm{~min}$. For slower heat-up rates, there is more time for "flow defects" to annihilate at lower temperatures, so that at $540^{\circ} \mathrm{C}$ flow does not occur so rapidly. In our experiment, the sample was heated up rapidly by being placed into a preheated apparatus.

${ }^{19}$ G. Q. Lu, E. Nygren, and M. J. Aziz, J. Appl. Phys. (to be published). 